\title{
CONVERGENCE OF DISCRETE APPROXIMATIONS FOR CONSTRAINED MINIMIZATION
}

\author{
B. D. CRAVEN ${ }^{1}$
}

(Received 17 February 1993)

\begin{abstract}
If a constrained minimization problem, under Lipschitz or uniformly continuous hypotheses on the functions, has a strict local minimum, then a small perturbation of the functions leads to a minimum of the perturbed problem, close to the unperturbed minimum. Conditions are given for the perturbed minimum point to be a Lipschitz function of a perturbation parameter. This is used to study convergence rate for a problem of continuous programming, when the variable is approximated by step-functions. Similar conclusions apply to computation of optimal control problems, approximating the control function by step-functions.
\end{abstract}

\section{Introduction}

In computing a constrained minimum, it is often necessary to approximate the given minimization problem. For example, a nonsmooth problem may be approximated by a smooth problem, or a minimization over a space of piecewise continuous functions (as is often required in optimal control) may be approximated by minimization over a prescribed sequence of step-functions. In numerical experiments such approximations often work very well (see for example [10]). This raises the question, what general properties are required of the functions in a minimization problem, in order that such approximation methods will converge to the minimum of the given problem, and at what rate?

As a simple example, let $f(x, q):=x_{+}^{2}+2 q x$ (where $x \in \mathbf{R}$, and $x_{+}:=x$ if $x \geq 0,0$ if $x<0$ ). If the parameter $q=0$, then $f(., 0)$ reaches an unconstrained minimum at 0 . If $q<0$, then $f(., q)$ reaches a minimum at $-q$; but if $q>0$, then $f(., q)$ does not reach any minimum. This function may be compared with $g(x, q):=x_{+}^{2}+\alpha(-x)_{+}^{2}+2 q x$, where $\alpha$ is small and positive. Unlike $f(., q), g(., q)$ has a strict minimum at 0 ; thus, for some $\theta(r)=\alpha r^{2}>0, g(x, 0) \geq \theta(r)$ whenever

\footnotetext{
'Mathematics Department, University of Melbourne, Parkville, Victoria 3052, Australia

(C) Australian Mathematical Society, 1994, Serial-fee code 0334-2700/94
} 
$|x-0|=r$. When $q>0, g(., q)$ is minimized at $-q /(2 \alpha)$. As $q$ varies from 0 , the minimum of $g(., q)$ remains in some neighbourhood of the minimum of $g(., 0)$; the size of this neighbourhood depends on $\alpha$. From this simple example, stability to perturbations should not be expected, if the given minimum is not strict.

\section{Approximating a problem}

Following the results in [4] and [5], consider a parametric family of constrained minimization problems:

$$
\text { Minimize }_{x} f(x, q) \text { subject to } p(x, q) \in K,
$$

in which $X$ and $Y$ are normed spaces, $K \subset Y$ is a closed convex cone, $x \in X$, and the parameter $q \in \mathbf{R}^{\ell}$. When $q=0$, let $f(x, 0)=f_{0}(x)$ and $p(x, 0)=p_{0}(x)$, where $f_{0}$ and $p_{0}$ are given functions. Assume that the functions $f(x, q)$ and $p(x, q)$ are uniformly continuous in $(x, q)$, when $x$ is in a bounded neighbourhood $\mathbf{N}^{\prime}(q)$ of $\Delta_{q}:=\{x \in X: p(x, q) \in K\}$, and $q \in \mathbf{N}$, a bounded neighbourhood of $0 \in \mathbf{R}^{\ell}$. (In finite dimensions, a compact set $\mathbf{E}$ may exist such that $(\forall q \in \mathbf{N}) \mathbf{N}^{\prime}(q) \subset \mathbf{E}$, and then it suffices to assume that the functions are continuous on $\mathbf{E} \times \mathbf{N}$. However, other validation must be found in infinite dimensions.) The following results also apply when $q$ is restricted to a discrete set, such as $\{1 / n: n=1,2,3, \ldots\}$. Let the point $a$ satisfy $p_{0}(a) \in K$. Define $\tilde{p}(.,$.$) by:$

$$
\tilde{p}(x, q):=p(x, q)-p(a, q)+p(a, 0) ;
$$

then $\tilde{p}(a, q)=\tilde{p}(a, 0)=p_{0}(a)$. Let $\Gamma_{q}:=\{x \in X: \tilde{p}(x, q) \in K\}$. For $a \in X$, let $B(a, r):=\{x \in X:\|x-a\| \leq r\}$. For $e \in E$, the distance $d(x, E):=\inf _{e \in E}\|d-e\|$. Let $A_{q}(r):=\Gamma_{q} \cap B(a, r)$, and $A_{q}^{\prime}(r):=\Gamma_{q} \cap\{x \in X:\|x-a\|=r\}$.

LEMMA 1. Assume $p(.,$.$) is uniformly continuous on bounded sets, and the point a$ satisfies $p_{0}(a) \in K$. Fix $r>0$; let $0<\epsilon<r$. Then, for sufficiently small $\|q\|$, each point of $A_{q}(r)$ is distant less than $\epsilon$ from $A_{0}(r)$.

PROOF. By construction, $a \in \Gamma$ and $a \in \Gamma_{q}$. For $0<\epsilon<r$, define

$$
\pi(\epsilon):=\inf \left\{d(p(x), K):\|x-a\| \leq r, d\left(x, A_{0}(r)\right) \geq \epsilon\right\} .
$$

Since $K$ is closed, if $\|x-a\| \leq r$ and $x \notin A_{0}(r)$, then $d\left(x, A_{0}(r)\right)>0, d(p(x), K)>$ 0 , and $\pi(\epsilon)>0$. By the uniform continuity, there is $\delta>0$ such that, if $\|q\|<\delta$, then $\|\tilde{p}(x, q)-\tilde{p}(x, 0)\|<\pi(\epsilon) / 3$, uniformly in $x$ in a neighbourhood of $A_{0}(r)$. Since also $\|\tilde{p}(a, q)-\tilde{p}(a, 0)\|<\pi(\epsilon) / 3$, it follows that $\tilde{p}(x, q)$ lies in a ball with centre 
$p(x, 0)$ and radius $2 \pi(\epsilon) / 3$, which is disjoint from the closed convex set $K$; hence $\tilde{p}(x, q) \notin K$. Taking the contrapositive,

$$
[\|x-a\| \leq r \text { and } \tilde{p}(x, q) \in K] \Rightarrow\left[\|x-a\| \leq r \text { and } d\left(x, A_{0}(r)\right) \leq \epsilon\right] .
$$

REMARKS. If $a$ is a boundary point of $\Gamma$, then there is a sequence $\left\{x_{j}\right\} \rightarrow a$, with each $x_{j} \notin \Gamma$; hence $\pi(\epsilon) \downarrow 0$ as $\epsilon \downarrow 0$. Otherwise, $\pi(\epsilon) \downarrow \pi(0)>0$, and $\delta$ does not tend to 0 as $\epsilon \downarrow 0$ in this proof. It suffices if the uniform continuity holds for $x$ in some bounded neighbourhood of $A_{0}(r)$.

LEMMA 2. Assume $p(.,$.$) is uniformly continuous on bounded sets, and the point a$ satisfies $p_{0}(a) \in K$. Fix $r>0$; let $0<\epsilon<r$. Then, for sufficiently small $\|q\|$, each point of $A_{q}^{\prime}(r)$ is distant less than $\epsilon$ from $A_{0}^{\prime}(r)$.

PROOF. The same proof holds with $A_{q}^{\prime}(r)$ replacing $A_{q}(r)$.

Now suppose that the point $a$ is a local minimum of the problem $(\mathrm{P}(0))$. For constant $\gamma>0$, define $F(x, q):=f(x, q)+\gamma\|x-a\|^{2}$. Then the point $a$ is a strict local minimum of $F(x, 0)$, subject to $p(x, 0) \in K$, meaning that

$$
\begin{array}{r}
(\forall \delta>0)(\exists \kappa>0)(\forall x, p(x, 0) \in K,\|x-a\|=\delta) \\
F(x, 0) \geq F(a, 0)+\kappa .
\end{array}
$$

The next lemma (based on [4]) shows that, because of the strict minimum, $F(., q)$ has a constrained minimum near to $a$, when $\|q\|$ is small.

LEMMA 3. Let $f(x, q)$ and $p(x, q)$ be uniformly continuous, for $x$ in a bounded neighbourhood of $A_{0}(r)$ and $q$ in a neighbourhood of 0 . When $q \neq 0$, assume that $F(., q)$ reaches a minimum on each closed bounded set. If $\|q\|$ is sufficiently small, then $F(x, q)$ reaches a minimum, subject to the constraint $\tilde{p}(x, q) \in K$, at a point $\bar{x}(q)$, where $\bar{x}(q) \rightarrow$ a as $\|q\| \rightarrow 0$.

PROOF. Let $0<r<\delta$; let $\epsilon>0$. Since $F(., 0)$ has a strict minimum at $a$, there is $\theta>0$ such that $F(\xi, 0) \geq F(a, 0)+4 \theta$ for all $\xi \in A_{0}^{\prime}(r)$. By the uniform continuity, $\left|F(x, 0)-F\left(x^{\prime}, 0\right)\right|<\theta$ and $|F(x, q)-F(x, 0)|<\theta$ whenever $\left\|x-x^{\prime}\right\|<\delta_{1}(\theta)$, say. If $\|q\|$ is sufficiently small, and $x \in A_{q}^{\prime}(r)$, then $d\left(x, A_{0}^{\prime}(r)\right) \leq \delta_{1}(\theta)$ by Lemma 2. So, for some $x^{\prime} \in A_{0}^{\prime}(r)$,

$$
\begin{aligned}
F(x, q)= & F(a, 0)+[F(x, q)-F(x, 0)]+\left[F(x, 0)-F\left(x^{\prime}, 0\right)\right] \\
& +\left[F\left(x^{\prime}, 0\right)-F(a, 0)\right] \\
\geq & F(a)-\theta-\theta+4 \theta .
\end{aligned}
$$


Similarly, if $\|q\|$ is sufficiently small, then

$$
F(a, q)=F(a, 0)+[F(a, q)-F(a, 0)] \leq F(a)+\theta .
$$

For $q \neq 0, F(., q)$ reaches a local minimum on the closed bounded set $A_{q}(r)$, say at $x=\bar{x}(q)$. Since $\left(\forall x \in A_{q}^{\prime}(r)\right) F(x, q)-F(a, q) \geq \theta>0, \bar{x}(q) \notin A_{q}^{\prime}(r)$. Hence $\bar{x}(q)$ is a local minimum of $F(., q)$ on $\Gamma_{q}$.

If now $r \downarrow 0$, then the corresponding $\theta \downarrow 0$ and $q \downarrow 0$. Since $\|\bar{x}(q)-a\|<r$, it follows that $\|\bar{x}(q)-a\| \rightarrow 0$ as $\|q\| \downarrow 0$.

\section{Lipschitz maps}

The results in Section 2 assume uniform continuity, but do not require any differentiability. Consider now the case where $p(.,$.$) is continuously (Fréchet) differentiable,$ and also the Robinson stability condition holds at the point $a$ when $q=0$, namely that $0 \in \operatorname{int}\left[\tilde{p}(a, 0)+\tilde{p}_{x}(a, 0)(X)-K\right]$. Then [8], for some constant $\kappa>0$, and all sufficiently small $\|q\|$,

$$
d(a,\{x: \tilde{p}(x, q) \in K\}) \leq \kappa d(0, \tilde{p}(a, q)-K)=\kappa \mathrm{O}(\|q\|),
$$

using differentiability. Hence, for some constant $\nu$, if $\|q\|<\nu \epsilon$, then $A_{q}(r)$ lies in an $\epsilon$ neighbourhood of $A_{0}(r)$. Otherwise expressed, $A_{q}(r)$ lies in a $\kappa^{-1}\|q\|$-neighbourhood of $A_{0}(r)$. A similar result holds also when $A_{q}(r)$ and $A_{0}(r)$ are replaced by $A_{q}^{\prime}(r)$ and $A_{0}^{\prime}(r)$ respectively. It is deduced by adjoining the additional constraint $\|x-a\|=r$; since this constraint is not generally active, it does not change the stability condition.

If $X$ and $Y$ are finite-dimensional, $p$ is Lipschitz (no longer continuously differentiable), the mapping $(x, q) \rightarrow \partial \tilde{p}(x, q)$ (the Clarke generalized Jacobian with respect to $x)$ is upper semicontinuous at $(a, 0)$, and the generalized stability condition holds:

$$
(\forall M \in \partial \tilde{p}(a, 0)) \quad 0 \in \operatorname{int}[\tilde{p}(a, 0)+M(X)-K],
$$

then Yen [12] has shown that $(\sharp)$ still holds. Hence, in this case also, it follows that $A_{q}^{\prime}(r)$ lies in a $\kappa^{-1}\|q\|$-neighbourhood of $A_{0}^{\prime}(r)$ for some constant $\kappa$.

LEMMA 4. Assume $p(.,$.$) is uniformly continuous on bounded sets, and Lipschitz;$ assume either the Robinson stability condition, or the generalized stability condition in finite dimensions. Let $F(.,$.$) be Lipschitz, with Lipschitz constant \kappa_{2}$. Let $F(., 0)$ reach a strict minimum at $x=a$, subject to $p(x, 0) \in K$, satisfying also the linear growth condition:

$$
F(x, 0)-F(a, 0) \geq \theta=\left(\kappa_{3} / 4\right) r \quad \text { when } \quad x \in A_{0}^{\prime}(r)
$$


for some constant $\kappa_{3}$. Then, for sufficiently small $\|q\|, F(x, q)$ reaches a minimum, subject to $\tilde{p}(x, q) \in K$, at $x=\bar{x}(q)$, satisfying (for some constant $\kappa_{4}$ ) the Lipschitz condition:

$$
\|\bar{x}(q)-a\| \leq \kappa_{4}\|q\|
$$

PROOF. From either stability hypothesis, there is a constant $\kappa_{1}$ so that $A_{q}^{\prime}(r)$ lies in a $\kappa_{1}\|q\|$-neighbourhood of $A_{0}^{\prime}(r)$, whenever $\|q\|$ is sufficiently small. This result is used in place of Lemma 2 in a modification, as follows, of the proof of Lemma 3.

If $x \in A_{0}^{\prime}(r)$ and $\|q\|=\left(\kappa_{2} \kappa_{1}\right)^{-1} \theta$, then

$$
d\left(x, A_{0}^{\prime}(r)\right) \leq d\left(A_{q}^{\prime}(r), A_{0}^{\prime}(r)\right) \leq \kappa_{2}^{-1} \theta .
$$

So, for some $x^{\prime} \in A_{0}^{\prime}(r), \kappa_{2}\left\|x-x^{\prime}\right\| \leq \theta$; hence

$$
F(x, q) \geq F(a, 0)-\theta-\theta+4 \theta .
$$

The rest of the proof is unchanged, showing that there is a minimum $\bar{x}(q)$ of $F(., q)$ on $\Gamma_{q}$, with $\|\bar{x}(q)-a\|<r$, where now

$$
\|q\|=\left(\kappa_{2} \kappa_{1}\right)^{-1} \theta=\left[\kappa_{3} /\left(4 \kappa_{1} \kappa_{2}\right)\right] r .
$$

Thus, setting $\kappa_{4}:=4 \kappa_{1} \kappa_{2} / \kappa_{3}$, the stated conclusion follows.

\section{Truncation in $\ell^{2}$}

To motivate the following, consider a constrained minimization problem with an objective function $\hat{f}(\xi)$, where the variable $\xi(.) \in L^{2}[0,1]$. Let $\xi$ be expanded in a Fourier-Walsh series, with expansion coefficients $x_{1}, x_{2}, \ldots$; then $x:=\left(x_{1}, x_{2}, \ldots\right) \in$ $\ell^{2}$. If the Fourier-Walsh series is truncated after the $k$ th term, this gives an approximation to $\xi\left(\right.$.) by a step-function, where $[0,1]$ is divided into $2^{k}$ subintervals, and the step-function is constant on each subinterval. Since $\xi=U x$ for some unitary transformation $U$, the convergence of such approximations to the solution of the original, infinite-dimensional, minimization problem may be studied equivalently in terms of $f(x):=\hat{f}(U x)$, where $x \in \ell^{2}$.

Consider then $X=\ell^{2}$. If $x=\left(x_{1}, x_{2}, \ldots\right) \in \ell^{2}$, denote by $x^{(n)}:=\left(x_{1}, \ldots, x_{n}\right.$, $0,0, \ldots)$ the truncation of $x$ to the first $n$ nonzero components. Similarly define $c^{(n)}$ from $c \in \ell^{2}$. Consider a minimization problem:

$$
\text { Minimize }_{x \in \ell^{2}} f(x) \text { subject to } p(x) \in K \text {. }
$$


Assume $P_{\infty}$ reaches a local minimum at $x=a$. Let $q:=1 / n(n=1,2, \ldots)$, $f(x, q):=f\left(x^{(n)}\right), p(x, q):=p\left(x^{(n)}\right)$, and $\tilde{p}$ defined from $p$ and $a$ as in Section 2 above. In some sense, the truncated problem:

$$
\text { Minimize }_{x \in \ell^{2}} f(x, 1 / n) \text { subject to } \tilde{p}(x, 1 / n) \in K
$$

approximates the given problem $P_{\infty}$.

In the particular case of a linear objective function $f(x):=\langle c, x\rangle$ with $c \in \ell^{2}$,

$$
|f(x, 0)-f(x, 1 / n)|=\left|\left\langle c, x-x^{(n)}\right\rangle\right|=\left|\left\langle c-c^{(n)}, x-x^{(n)}\right\rangle\right| \leq \theta_{n}\|x\|,
$$

where $\theta_{n}:=\sup _{j>n}\left|c_{j}\right| \rightarrow 0$ as $n \rightarrow \infty$. So the desired uniform approximation occurs on norm-bounded sets. For a quadratic function, uniform approximation is attained only by restricting $x$ to a subspace of $\ell^{2}$. Let $0<\left\{b_{j}\right\} \rightarrow \infty$; let $w_{j}:=b_{j}^{2}$; restrict $x$ so that the weighted norm $\|x\|^{w}:=\left[\sum w_{j} x_{j}^{2}\right]^{1 / 2}<\infty$. Then

$$
\begin{aligned}
\langle x, x\rangle-\left\langle x^{(n)}, x^{(n)}\right\rangle & =\langle z, t\rangle \text { where } t:=x-x^{(n)} \text { and } z:=2 x-t \\
|\langle z, t\rangle| & \leq\left[\sum_{j>n}\left(b_{i}^{-2} b_{i} z_{i}\right)^{2} \cdot \sum_{j}\left(b_{j} t_{j}\right)^{2}\right]^{1 / 2} \\
& \leq \theta_{n}\left[\sum\left(b_{i} z_{i}\right)^{2} \cdot \sum_{j}\left(b_{j} t_{j}\right)^{2}\right]^{1 / 2} \\
& =\theta_{n} \cdot\|z\|^{w} \cdot\|t\|^{w} \leq \theta_{n}(3\|x\|)\|x\|,
\end{aligned}
$$

where $\theta_{n}:=\sup _{j>n} w_{j} \rightarrow 0$ as $n \rightarrow \infty$.

Consider now a function $\xi($.$) in a suitable subspace S$ of $L^{2}[0,1]$, and $x \in \ell^{2}$ the sequence of the Fourier-Walsh coefficients for $\xi$. Assume that $\|x\|^{w}<\infty$; this restricts high-frequency oscillations of $x($.$) , but does not restrict \xi($.$) to a finite-$ dimensional subspace. Let $f(x):=<x, x>$. From the previous paragraph, $f\left(x^{(n)}\right)$ uniformly approximates $f(x)$ on norm-bounded subsets. Now $x^{(n)}$ is the transform of a step-function, $\xi_{n}\left(\right.$.) say on $[0,1]$. Let $\varphi(\xi):=\int_{0}^{1} \xi(s)^{2} d s$; then $\varphi(\xi)=f(x)$. Hence $\varphi\left(\xi_{n}\right)$ uniformly approximates $\varphi(\xi)$ on bounded subsets of $\mathbf{S}$. A similar construction applies to a functional $\varphi(\xi):=\int h(\xi(s), s) d s$, if the transform $f(x)$ satisfies $\mid f(x)-$ $f\left(x^{(n)} \mid \leq\left\langle c(x), x-x^{(n)}\right\rangle\right.$, where $\|c(x)\| \leq$ const $\|x\|$.

\section{Constrained minimum}

Consider a constrained continuous programming problem:

Minimize $f(x)$ subject to $p(x) \in K$, 
where $f(x):=\int_{l} h(x(t), t) d t, l=[0,1], x(.) \in L^{2}[0,1], p(x)()=.k(x(), t$. $(t \in I), K$ is a cone of nonnegative functions on 1 . Denote by $x_{n}($.$) a step-function$ approximation to $x($.), obtained by truncating $I$ into $n$ subintervals (where $n$ is a power of 2.) Denote by $\left(\mathrm{CP}_{n}\right)$ the approximated problem, obtained by replacing $x($.$) by x_{n}($. in (CP).

THEOREM 1. For problem (CP), let $x($.$) lie in a subspace \mathbf{S}$ of $L^{2}(I)$, such that uniform approximation of $(\mathrm{CP})$ by step-functions $x_{n}($.$) holds:$

$$
\begin{aligned}
(\forall \epsilon>0, r>0) & (\exists n)(\forall x \in \mathbf{S},\|x\|<r) \\
& \left|f\left(x_{n}\right)-f(x)\right|<\epsilon, \quad\left\|p\left(x_{n}\right)-p(x)\right\|<\epsilon .
\end{aligned}
$$

Assume that $(\mathrm{CP})$ reaches a minimum at $x()=.\bar{x}($.$) . Then \left(\mathrm{CP}_{n}\right)$ reaches a local minimum at a point $\bar{x}_{n}$, where $\bar{x}_{n} \rightarrow \bar{x}$ as $n \rightarrow \infty$.

If in addition, the functions $h$ and $k$ are Fréchet differentiable and the Robinson stability condition holds, and $f$ satisfies the linear growth condition, then there is a Lipschitz constant $\kappa$ so that $\left\|\bar{x}_{n}-\bar{x}\right\| \leq \kappa / n$.

PROOF. The approximated problem $\left(\mathrm{CP}_{n}\right)$ involves only finitely many (say $r$ ) variables, hence $f(x)$, with $x$ restricted to $\mathrm{S}$, reaches a minimum on each closed bounded subset. From Lemma 3 , problem $\left(\mathrm{CP}_{n}\right)$ reaches a minimum at a point, $\bar{x}_{n}$ say, for which $\left\|\bar{x}_{n}-\bar{x}\right\| \rightarrow 0$ as $n \rightarrow \infty$.

The remaining conclusion follows from Lemma 4.

REMARKS. A similar result holds for various optimal control problems. Some cases when the uniform approximation property holds are detailed in Section 4. See [5] for a discussion of when the linear growth condition holds for $f($.$) . If linear growth is$ weakened to quadratic growth (see for example [1],[2]), then the Lipschitz conclusion of Lemma 4 is weakened to a Hölder condition: $\|\bar{x}(q)-a\| \leq \kappa_{4}\|q\|^{1 / 2}$, and then in Theorem $1,\left\|\bar{x}_{n}-\bar{x}\right\| \leq \kappa / n^{1 / 2}$.

\section{Optimal control}

Consider an optimal control problem:

$$
\begin{gathered}
\text { Minimize } J(u)=\int_{0}^{T} f_{0}(x(t), u(t), t) d t+\Phi(x(T)) \text { subject to } \\
x(0)=x_{0} ; \quad d x(t) / d t=m(x(t), u(t), t) \quad(0 \leq t \leq T) \\
u(t) \in \Gamma(t) \quad(0 \leq t \leq T) .
\end{gathered}
$$

Here, $x(t)$ is the state function, $u(t)$ is the control function; $f(., .,),. \Phi($.$) and m(., .,$. are continuously differentiable (and hence Lipschitz) functions, and $\Gamma(t)$ is a convex 
set, depending smoothly on $t$. The objective function can be written as $J(u)$, since the differential equation for $d x(t) / d t$ determines $x($.) uniquely as a Lipschitz function of $u($.). Assume (as commonly happens in applications) that (OC) reaches an optimum, with the optimal control $\bar{u}($.) a piecewise-continuous function.

The conclusions of Theorem 1 apply also to the optimal control problem (OC), now replacing $x($.$) of (CP) by the control function u($.$) of (\mathrm{OC})$, taking the subspace $\mathbf{S}$ in Theorem 1 to be a subspace of piecewise-continuous functions such that uniform approximation by step-functions holds, and assuming the Robinson stability condition. Then Theorem 1 shows that approximating $u\left(\right.$.) by a step-function $u_{n}($.) (whereby the interval $[0, T]$ is divided into $2^{n}$ equal subintervals, with $u_{n}($.$) constant on each),$ leads to an approximate optimum function $\bar{u}_{n}($.$) , such that \left\|\bar{u}_{n}()-.\bar{u}().\right\|<\kappa / n$ in an appropriate norm, as $n \rightarrow \infty$. There is an obvious extension when $2^{n}$ is replaced by another integer $r$, with $r \rightarrow \infty$.

Such step-function approximations to the control function have proved very successful in computational practice-see [6], [9], [10], [11], and other papers cited in these references. The present results show that such approximations will work well under quite general conditions. An open question remains, as to what improvement in convergence might be had, by using spline approximations of higher order.

\section{Convergence under different hypotheses}

Consider the minimization problem in the form:

Minimize $f(x)$ subject to $x \in \Gamma$,

where $\Gamma$ is a closed subset of $\ell^{2}$, and $f$ is Lipschitz, with Lipschitz constant $\kappa$. Assume that a minimum is reached at $x=\bar{x}$. Denote by $P_{n}$ the projection that takes $x=$ $\left(x_{1}, x_{2}, \ldots\right) \in \ell^{2}$ to $P_{n} x=\left(x_{1}, x_{2}, \ldots, x_{n}, 0,0, \ldots\right)$. Note that $\Gamma \cap P_{n}\left(\ell^{2}\right)=P_{n} \Gamma_{n}$ for some maximal subset $T_{n} \subset \Gamma$; usually $\Gamma_{n} \neq \Gamma$.

Assume that the approximated problem, obtained by restricting $x$ to $P_{n}\left(\ell^{2}\right)$, reaches a minimum at $\tilde{x}_{[n]}$. Denote by $\Delta_{n}$ the feasible set for this approximated problem.

Consider the following hypotheses:

(H1): $(\forall x \in \Gamma) d\left(P_{n} x, \Gamma \cap P_{n}\left(\ell^{2}\right)\right) \rightarrow 0$ as $n \rightarrow \infty$.

(H2): If $E$ is a bounded set containing $\bar{x}$, and $\epsilon^{\prime}>0$, then for sufficiently large $n$, $E \cap \Delta_{n}$ is contained in an $\epsilon^{\prime}$-neighbourhood of $E \cap \Gamma$, and $E \cap \Gamma$ is contained in an $\epsilon^{\prime}$-neighbourhood of $E \cap \Delta_{n}$.

(H3): The global minimum $\bar{x}$ of the given problem is strict.

THEOREM 2. If $f($.$) reaches a minimum over \Gamma$ at $\bar{x}$, and reaches a minimum over $\Gamma \cap P_{n}\left(\ell^{2}\right)$ at $\tilde{x}_{[n]}$, and if $(\mathrm{H} 1)$ holds, then $\left\{f\left(\tilde{x}_{[n]}\right)\right\} \downarrow f(\bar{x})$. 
PROOF. Let $\tau_{n}:=d\left(P_{n} \bar{x}, \Gamma \cap P_{n}\left(\ell^{2}\right)\right)$. By (H1), $\tau_{n} \rightarrow 0$ as $n \rightarrow \infty$. There is a point $x_{n} \in \Gamma \cap P_{n}\left(\ell^{2}\right)$ such that $\left\|P_{n} \bar{x}-x_{n}\right\| \leq 2 \tau_{n}$. Then

$$
\begin{aligned}
f(\bar{x}) \leq & f\left(\tilde{x}_{[n]}\right) \leq f\left(x_{n}\right) \\
& \leq f(\bar{x})+\left|f(\bar{x})-f\left(\tilde{x}_{[n]}\right)\right|+\left|f\left(\tilde{x}_{[n]}\right)-f\left(x_{n}\right)\right| \leq f(\bar{x})+\kappa \epsilon+\kappa\left(2 \tau_{n}\right) .
\end{aligned}
$$

The convergence is monotone since the feasible set is expanding.

RemarKs. The sequence $\left\{f\left(\tilde{x}_{[n]}\right)\right\}$ decreases, but not always to $f(\bar{x})$. But the hypothesis (H1), to ensure convergence to $f(\bar{x})$, is very strong. See Lemma 1 for conditions when $(\mathrm{H} 2)$ is satisfied.

To obtain a computable problem, a further approximation is needed, to replace $\Gamma$ by $\Delta_{n}$.

THEOREM 3. Let $f($.$) be minimized over \Gamma$ at $\bar{x}$; let $(\mathrm{H} 1)$ and $(\mathrm{H} 3)$ hold; let $\Delta_{n}$ be bounded closed for each $n$. Then there exists a minimum point $w_{(n)}$ for $f($.$) over$ $\Gamma \cap P_{n}\left(\ell^{2}\right)$, such that $\left\{w_{(n)}\right\} \rightarrow \bar{x}$, and $\left\{f\left(w_{(n)}\right)\right\} \rightarrow f(\bar{x})$.

Proof. Let $\epsilon>0$. From (H3), $f(x) \geq f(\bar{x})+7 \kappa \epsilon^{\prime \prime}$ whenever $x \in \Gamma$ and $d(\bar{x}, x)=$ $r\left(\epsilon^{\prime \prime}\right)$, for some $0 \neq r\left(\epsilon^{\prime \prime}\right) \downarrow 0$ as $\epsilon^{\prime \prime} \downarrow 0$. If $E$ is a bounded set containing $\bar{x}$, then

$$
\begin{aligned}
(\forall \epsilon>0)(\exists n(\epsilon, E))(\forall n \geq & n(\epsilon, E))(\forall x \in E) \\
& \left|f(x)-f\left(P_{n} x\right)\right| \leq \kappa\left|x-P_{n} x\right| \leq \kappa \epsilon .
\end{aligned}
$$

If $E=B\left(\bar{x}, r_{0}\right)$, for some $r_{0}>0$, then $\left|f(x)-f\left(P_{n} x\right)\right| \leq \kappa \epsilon$ for all $n$ sufficiently large. From (H1), $\left\|P_{n} \bar{x}-v_{n}\right\|<\epsilon$ when $v_{n} \in \Gamma \cap P_{n}\left(\ell^{2}\right)$. If $w \in \Delta_{n} \cap \partial\left(P_{n} B(\bar{x}, r(\epsilon))\right)$ and $\epsilon^{\prime}$ in (H2) is chosen sufficiently small, there is $w^{\prime} \in \Gamma \cap \partial\left(P_{n} B(\bar{x}, r(\epsilon))\right)$ for which $\left\|w-w^{\prime}\right\|<\epsilon$, and there is $y \in \Delta_{n} \cap P_{n}\left(\ell^{2}\right)$ such that $\left\|y-v_{n}\right\|<\epsilon$. Since $f$ has Lipschitz constant $\kappa, f(y) \leq f\left(v_{n}\right)+\kappa \epsilon \leq f\left(P_{n} \bar{x}\right)+\kappa \epsilon+\kappa \epsilon$. Also there is $z^{\prime}$ orthogonal to $P_{n}\left(\ell^{2}\right)$ such that $w^{\prime}+z^{\prime} \in \Gamma \cap \partial B(\bar{x}, r(\epsilon))$. From (H3), $f\left(w^{\prime}+z^{\prime}\right) \geq f(\bar{x})+7 \kappa \epsilon ;$ since $w^{\prime}=P_{n}\left(w^{\prime}+z^{\prime}\right), f\left(w^{\prime}\right) \geq f(\bar{x})+6 \kappa \epsilon$. Hence

$$
f(w) \geq f(\bar{x})+6 \kappa \epsilon-\kappa\left\|w-w^{\prime}\right\| \geq f(\bar{x})+5 \kappa \epsilon \geq f\left(P_{n} \bar{x}\right)-\kappa \epsilon+5 \kappa \epsilon .
$$

Thus $w \neq y$; so no point $w \in \Delta_{n} \cap \partial\left(P_{n} B(\bar{x}, r(\epsilon))\right)$ can minimize $f($.$) over \Delta_{n} \cap$ $P_{n} B(\bar{x}, r(\epsilon))$. Such a minimum point exists, say $w^{(n)}$, since the set is bounded closed in finite dimensions. Since the constraint imposed by the boundary of the set is thus not binding, the point $w^{(n)}$ minimizes $f\left(\right.$.) over $\Delta_{n} \cap P_{n}\left(\ell^{2}\right)$. By construction, $\left\|w^{(n)}-\bar{x}\right\|$. By choosing suitable sequences of $\epsilon \downarrow 0$ and corresponding $n(\epsilon) \rightarrow \infty$, there follows $\left\{w^{(n)}\right\} \rightarrow \bar{x}$. Since $f($.$) is continuous, \left\{f\left(w^{(n)}\right)\right\} \rightarrow f(\bar{x})$. 
REMARK. If a given constraint holds for infinitely many points (for example, $(\forall t) \xi(t)$ $\geq 0$ in the original problem), it must be approximated computationally by a constraint which holds only for finitely many points. Assuming suitable continuity, $\Delta_{n}$ may be bracketed by two perturbed versions of the given feasible set. (For example, a constraint $|\xi(t)| \leq 1$ may be bracketed by two constraints $\left|\xi^{*}(t)\right| \geq 1-\epsilon$ and $\left|\xi^{*}(t)\right| \leq 1+\epsilon$, where $\xi^{*}(t)$ approximate $\xi(t)$ by considering only a finite grid of points.) If the original problem is stable to small perturbations of constraints (say of $g(x) \leq 0$ to $g(x) \leq \epsilon$ ), then the usual perturbation theory (see for example [8], [3]) may be applied. Consequently, this additional approximation will only have a small effect on the optimal objective function, when $\epsilon$ is small.

\section{Acknowledgement}

Some of this research was done while visiting the University of Poitiers. Section 7 was done while visiting the University of Western Australia.

\section{References}

[1] W. Alt, "Stability of solutions for a class of nonlinear cone constrained optimization problems", Numer. Funct. Anal. Appl. 10 (1989) 1053-1064 and 1064-1076.

[2] W. Alt, Stability of solutions and the Lagrange-Newton method for nonlinear optimization and optimal control problems (Habilitationsschrift, Universität Bayreuth, 1990).

[3] B. D. Craven, "Perturbed minimization, with constraints adjoined or deleted", Optimization 14 (1983) 23-26.

[4] B. D. Craven, "Nondifferentiable optimization by smooth approximations", Optimization 17 (1986) 3-17.

[5] B. D. Craven and R. Janin, "On stability in constrained optimization", submitted for publication, 1992.

[6] L. S. Jennings, M. E. Fisher, K. L. Teo and C. J. Goh, MISER3, Optimal Control Software, Theory and User Manual (1990).

[7] F. Lempio and H. Maurer, "Differential stability in infinite-dimensional nonlinear programming", Appl. Math. Optim. 6 (1980) 139-152.

[8] S. M. Robinson, "Stability theory for systems of inequalities, Part II: Differentiable nonlinear systems", SIAM J. Numer. Anal. 13 (1976) 497-513.

[9] K. L. Teo and C. J. Goh, "A computational method for combined optimal parameter selection and optimal control problems with general constraints", J. Austral. Math. Soc. Ser. B 30 (1989) 350-364.

[10] K. L. Teo, C. J. Goh and K. H. Wong, A unified computational approach to optimal control problems (Longman Scientific and Technical, 1991).

[11] K. L. Teo and R. S. Womersley, "A control parametrization algorithm for optimal control problems involving linear systems and linear terminal inequality constraints", Numer. Funct. Anal. Opt. 6 (1983) 291-313.

[12] N. D. Yen, "Stability of the solution set of perturbed nonsmooth inequality systems", J. Optim. Theor. Appl., to appear. 